\title{
A growing microcolony can survive and support persistent propagation of virulent phages
}

\author{
Rasmus Skytte Eriksen ${ }^{\mathrm{a}}$, Sine L. Svenningsen ${ }^{\mathrm{b}, 1}$, Kim Sneppen $^{\mathrm{a}}$, and Namiko Mitarai ${ }^{\mathrm{a}, 1}$ \\ a Niels Bohr Institute, University of Copenhagen, DK-2100 Copenhagen, Denmark; and ' Department of Biology, University of Copenhagen, DK-2200 \\ Copenhagen, Denmark
}

Edited by Bruce R. Levin, Emory University, Atlanta, GA, and approved November 27, 2017 (received for review May 30, 2017)

\begin{abstract}
Bacteria form colonies and secrete extracellular polymeric substances that surround the individual cells. These spatial structures are often associated with collaboration and quorum sensing between the bacteria. Here we investigate the mutual protection provided by spherical growth of a monoclonal colony during exposure to phages that proliferate on its surface. As a proof of concept we exposed growing colonies of Escherichia coli to a virulent mutant of phage P1. When the colony consists of less than $\sim 50,000$ members it is eliminated, while larger initial colonies allow long-term survival of both phage-resistant mutants and, importantly, colonies of mostly phage-sensitive members. A mathematical model predicts that colonies formed solely by phagesensitive bacteria can survive because the growth of bacteria throughout the colony exceeds the killing of bacteria on the surface and pinpoints how the critical colony size depends on key parameters in the phage infection cycle.
\end{abstract}

bacteria | spatial structure | coexistence | endemic | predator-prey

V irulent phages are ubiquitous, yet seemingly at odds with their own survival. As they kill their susceptible hosts, they suppress these to low population levels (1-3), creating a fragile and hugely competitive environment for themselves (4-6). By contrast, temperate phages have the option to lysogenize their host and thereby preserve their DNA through periods of low host availability $(7,8)$. This apparent advantage of temperate over virulent phages suggests the need for a more fine-grained understanding of virulence.

Notably, the virulent phage species present a quite diverse set of characteristics for their killing. Even under identical conditions, different species have average burst sizes that vary by more than a factor of 10 and have latency times that vary by a factor of 4 (9). Some virulent phages such as T3 and T7 are known to form particularly large plaques (10), while for example T4 forms rather small plaques due to lysis inhibition $(10,11)$, reflecting the variation in the "art of killing" (12).

Here we explore a demographic aspect of virulence, namely how a virulent phage propagates in a growing bacterial colony. Our investigation is inspired by visual inspection of the plaque formed by a virulent phage in Fig. 1 . The picture shows a "clear" plaque, caused by one $\mathrm{P} 1_{\text {vir }}$ phage that was introduced onto a bacterial lawn consisting of Escherichia coli cells. A nearly clear region surrounds the initial infection at the center, but one can also observe increasingly larger faint colonies as one moves away from this center. Outside the plaque, the agar contains a dense collection of colonies that have grown to stationary phase without the influence of phages. Bacteria in the periphery of the plaque encountered phages relatively later, after each bacterial cell had grown to form a microcolony (13). The origin of the faint colonies at the plaque periphery has not been fully understood (14).

This paper explores the fate of growing bacterial colonies that are exposed to phages at various time points during their growth. A previously proposed scenario (14) discussed that cells in the center of a large microcolony may enter stationary phase and become unable to support the production of progeny phages.
Although such a protection mechanism is certainly expected, recent work showed that the growth of even quite large E. coli colonies $\left(\sim 10^{7}\right.$ cells $)$ mimics the normal exponential growth of individual $E$. coli cells in liquid medium (15). This finding indicates that nutrients diffuse extensively through the colony. By contrast, phages may well adsorb in large numbers to susceptible cells on the colony surface and therefore only rarely reach deeper layers of the colony. Thus, we hypothesize that the continued growth from the inside of a sufficiently large colony could overwhelm killing on the colony surface. In combination with the phage-refractory state of stationary-phase cells, the result would be long-term survival of phage-sensitive cells inside the colony.

Here, we first construct a model of growing cells and infecting phages to numerically simulate the phage attack on a growing microcolony. The simulation suggests a sharp transition from elimination of the microcolony to its continued growth as the microcolony size at the time of the first phage encounter increases. We then show that the model prediction is consistent with an experiment by infecting $E$. coli colonies of different sizes with $\mathrm{P} 1_{\text {vir }}$ and monitoring their fate. Our study demonstrates that a sufficiently large colony indeed provides a refuge against $\mathrm{P} 1_{\text {vir. }}$. The refuge counteracts the phages' ability to control bacterial biomass (6) and thereby allows the bacteria to circumvent the famous kill-the-winner (16) feature of phage predation in wellmixed environments.

\section{Results}

Model. We simulate microcolony growth by modeling each cell as a sphere, and each cell grows and divides exponentially with a minimum doubling time of $\sim 20 \mathrm{~min}$. The repulsive force

\section{Significance}

Bacteria are repeatedly exposed to an excess of phages and carry evidence of this in terms of multiple defense mechanisms encoded in their genome. In addition to molecular mechanisms, bacteria may exploit the defense of spatial refuges. Here we demonstrate how bacteria can limit the impact of a virulent phage attack by growing as a colony which exposes only its surface to phages. We identify a critical size of the initial colony, below which the phages entirely eliminate the colony and above which the colony continues to grow despite the presence of phages. Our study suggests that coexistence of phages and bacteria is strongly influenced by the spatial composition of microcolonies of susceptible bacteria.

Author contributions: R.S.E., S.L.S., K.S., and N.M. designed research, performed research, analyzed data, and wrote the paper.

The authors declare no conflict of interest.

This article is a PNAS Direct Submission.

Published under the PNAS license.

${ }^{1}$ To whom correspondence may be addressed. Email: mitarai@nbi.ku.dk or sls@bio.ku.dk This article contains supporting information online at www.pnas.org/lookup/suppl/doi:10. 1073/pnas.1708954115/-/DCSupplemental. 


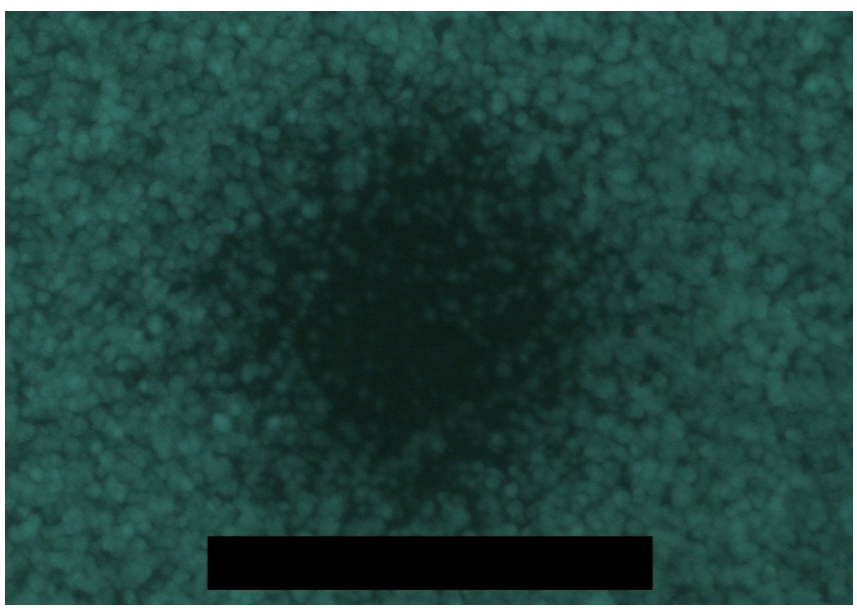

Fig. 1. Plaque formed by $\mathrm{P} 1_{\text {vir. }}$ Green spheres are microcolonies of $E$. coli expressing green fluorescent protein. Note the faint colonies, which are seen across the plaque although less in its central region. (Scale bar: $300 \mu \mathrm{m}$.)

between the cells provides an overall spherical growth of the microcolony consisting of densely packed bacteria. At a certain time, we let the surface of a grown microcolony be surrounded by $\sim 2,700$ phages and observe whether the phages kill all the cells or whether the cells can outgrow the ongoing lysis by the phages. The simulated microcolonies are rather small, and the explicit modeling of nutrient gradients inside a microcolony gave only minor growth rate differences across the colony (SI Appendix, section 3). Therefore, we here present the results from simulations that assume a constant nutrient level across the microcolony. The detailed simulation protocol is described in Methods and SI Appendix, section 1, with the default parameter values with dimensions (mostly measured in liquid culture conditions) in Table S1.

Fig. 2 illustrates model simulations with uninfected bacterial cells shown as blue spheres. Bacteria infected by phages are colored from yellow to red, where the ones closest to lysis are assigned the most reddish color. Each lysis event is modeled by replacement of the bacterium with 400 point-like phage particles at the end of the latency period. The progeny phages (not shown in the image) are allowed to diffuse and infect other bacteria. In this simulation, we assume that a phage particle adsorbs to a cell as soon as they overlap, mimicking the diffusion-limited scenario of adsorption. The phages adsorb equally well to uninfected cells and those that are already infected by another phage(s). We later study the effect of a smaller adsorption rate on microcolony growth.

In Fig. 2, Upper row, the first attack occurs at $3.25 \mathrm{~h}$ of growth, when the colony has a size of $\sim 220$ members. Fig. 2, Lower row depicts a phage attack at $3.75 \mathrm{~h}$ where the colony size has reached $\sim 500$ members. The smaller colony is eventually entirely eliminated, which would leave the fate of the phage particles to the chance that they reach another colony before they decay. In contrast, when the phages attack the larger colony, it survives and grows with phages persisting on the infected surface. The simulation showed that the distance phages can penetrate into the microcolony, $\Delta R$, is roughly constant over time when the microcolony size is above the threshold of killing (SI Appendix, section 4). For the parameters used in Fig. 2, phages can infect about two layers of cells. This limited penetration is observed because new phages released by lysed cells on the surface mostly adsorb to the cells in their immediate vicinity instead of diffusing deeper into the microcolony. The result is a high multiplicity of infection of the surface cells (cf. ref. 17) with the cells closer to bursting located nearer to the surface (Fig. 2) and preservation of uninfected cells closer to the center.

Fig. 3 examines the predictions of the model. Fig. $3 A$ shows the final growth rate of a colony as a function of the time at which it first encountered the phages. Indeed, the model predicts a sharp transition from extinction to persistent growth, when the size of the microcolony has surpassed a lower critical threshold. It is also apparent that if the first infection occurs sufficiently late to allow colony survival, the colony will reach a size where the growth penalty associated with infections at its surface becomes relatively small. Note that the growth rate is measured while the nutrient depletion is assumed to be negligible. In reality this assumption will ultimately break down when the inner part of the colony becomes increasingly shielded from available nutrients. The lack of nutrients will, however, also prevent or at least reduce phage proliferation $(10,14)$. Thus, if the colony is above the survival threshold, it is predicted to eventually maintain a large number of living sensitive cells in the center.

Assuming a constant penetration depth $\Delta R$ of the phages into a growing colony allows us to understand the steep sizedependent transition in microcolony fate. Suppose that a spherical microcolony of radius $R(t)$ at time $t$ consists of an inner core of exponentially growing uninfected cells with growth rate of biomass $g$ and an infected surface layer of a constant thickness $\Delta R$. We further assume that infected cells will burst with a rate $1 / \tau_{L}$, with $\tau_{L}$ being the latency time of the phage burst. This leads to the equation for the total volume $V(t)=\frac{4 \pi}{3} R(t)^{3}$ to be

$$
\frac{d V(t)}{d t}=\frac{4}{3} \pi\left[g(R(t)-\Delta R)^{3}-\frac{1}{\tau_{L}}\left[R(t)^{3}-(R(t)-\Delta R)^{3}\right]\right],
$$

where the first term in the brackets depicts the growth of the uninfected cells in the inner core, while the second term describes the lysis of the outer infected layer. Clearly, the growth of the total volume $d V(t) / d t>0$ requires large enough $R(t)$ compared with $\Delta R$. The critical threshold $R_{c}$ for the initial size $R(0)$ is determined by $d V(0) / d t=0$. The solution is given in $S I$ Appendix, Eq. $\mathbf{S 7}$ and fits well with the simulation (Fig. $3 B$ ). If we approximate the solution for a small penetration depth case $\left(\Delta R \ll R_{c}\right)$, we get $R_{c}=3\left[1+\left(g \tau_{L}\right)^{-1}\right] \Delta R$ (derivation in $S I$ Appendix, section 5), depicting that $R_{c}$ increases with $\Delta R$ and that a smaller burst rate $1 / \tau_{L}$ compared with the cell growth rate $g$ decreases $R_{c}$.

$R_{c}$ depends on the various parameters as demonstrated in Fig. $3 B-D$. $R_{c}$ grows roughly linearly with $1 / \tau_{L}$ (Fig. $3 B$ ), because the latency time defines the timespan where an infected cell can

\section{$\mathrm{T}=3.25 \mathrm{~h} \quad \mathrm{~T}=4.58 \mathrm{~h} \quad \mathrm{~T}=5.92 \mathrm{~h} \quad \mathrm{~T}=7.25 \mathrm{~h}$
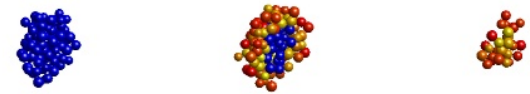

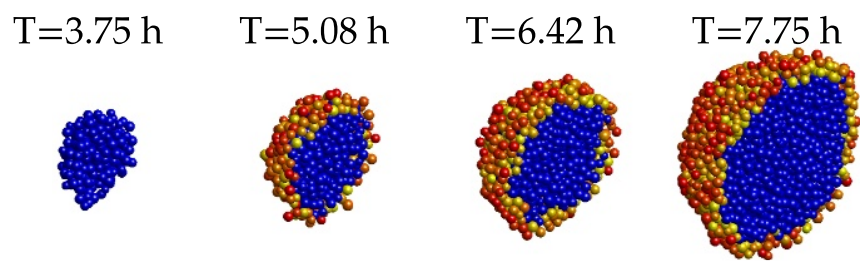

Fig. 2. Snapshots of simulations with half of the microcolonies presented. (Upper row) The case of a relatively early phage attack (infection at $3.25 \mathrm{~h}$ ), which results in elimination of the microcolony. (Lower row) The history of a slightly later attack (infection at $3.75 \mathrm{~h}$ ), which results in continued growth of the microcolony. 

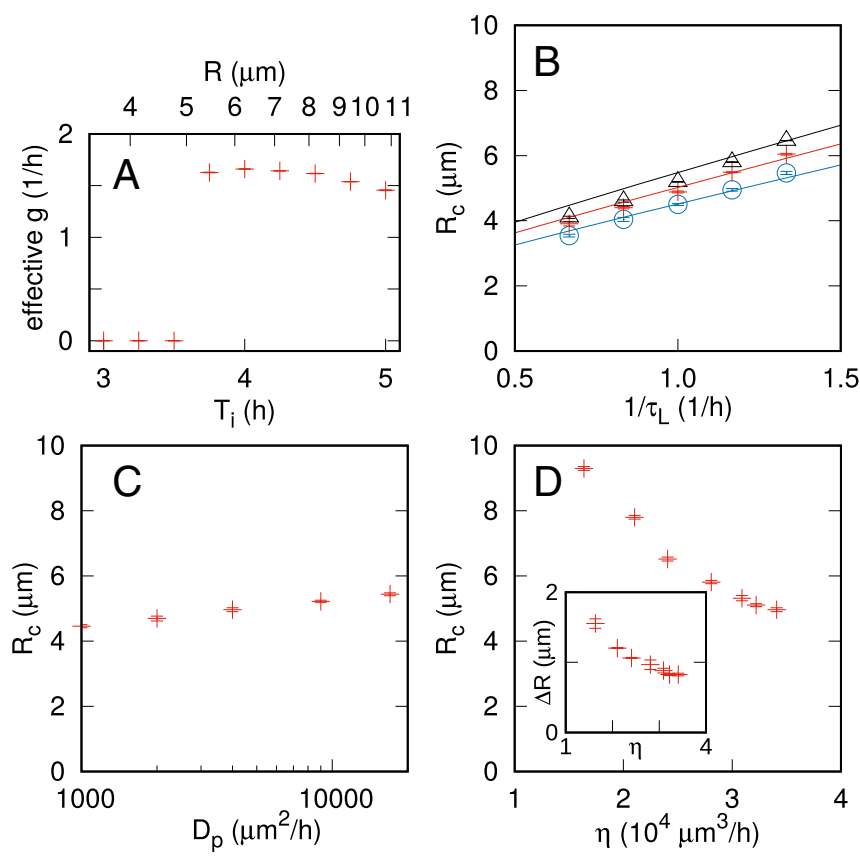

Fig. 3. Model predictions. $(A)$ Long-term growth rate of phage-infected colony as a function of initial exposure time $\left(T_{i}\right)$. The effective growth rate $g$ was determined by fitting the analytical solution (SI Appendix, Eq. S9) of the linearized version of the model equation to the simulation time traces of the radius $R(t)$ (see $S I$ Appendix, section 5 for details). The radius of the colony at the initial exposure time is shown on the top horizontal axis. $(B)$ The critical radius $R_{C}$ as a function of latency time $\tau_{L}$ for burst sizes of 100 (open blue circles), 400 (red crosses), and 1,000 (open black triangles). The fit of SI Appendix, Eq. S7 to each data point is shown as a solid line, with the fitting parameter $\Delta R=1.26 \mu \mathrm{m}, 1.40 \mu \mathrm{m}$, and $1.53 \mu \mathrm{m}$, respectively. $(C) R_{c}$ as a function of the phage diffusion constant $D_{P}$. (D) $R_{C}$ as a function of the phage adsorption rate $\eta$. $D$, Inset shows the phage penetration depth $\Delta R$. The SEM is shown as error bars.

continue to shield the uninfected core by adsorbing additional phage particles until it undergoes lysis. The burst size affects $\Delta R$ and hence $R_{c}$, but changing the burst size from 100 to 1,000 increases the critical radius $R_{c}$ only by about one cell layer with the given parameter set (Fig. $3 B$ ). A larger phage diffusion constant $D_{p}$ only marginally increases the critical radius (Fig. 3C), because the phages are hindered from reaching the core cells by adsorption to the infected surface cells regardless of the magnitude of the diffusion constant.

Reducing the adsorption rate $\eta$ from the diffusion-limited case causes a substantial increase in the critical radius $R_{c}$. To simulate small $\eta$ we allowed the phage particles that overlap a cell to be repelled away and introduced a finite rate $\gamma$ with which infection can occur while there is overlap. Reducing $\gamma$ parameterizes a reduction of the phage adsorption rate $\eta$ from the diffusionlimited value. The reduction of $\eta$ dramatically increases the critical radius $R_{c}$ (Fig. 3D). Note that the values of $\eta$ shown are determined in the well-mixed condition (SI Appendix, section 2), and the actual adsorption events to densely packed hosts cannot be simply given by $\eta$ multiplied by the densities of the phage and bacteria. The reduction of $R_{c}$ is caused by an increased penetration depth $\Delta R$ as phages can diffuse deeper into the colony core before they adsorb to a cell, demonstrating the importance of the high multiplicity of infection of cells at the surface for the protection of the core.

Infection of $\boldsymbol{E}$. coli Colonies with $\mathbf{P 1}_{\text {vir }}$. To test the above predictions in the laboratory, we performed infections of E. coli microcolonies with a virulent mutant of the phage $\mathrm{P} 1, \mathrm{P} 1_{\text {vir }}$
(18). This phage has a fairly long latency time of $60 \mathrm{~min}$ for $E$. coli growing in rich medium (9), thus facilitating a moderately small value of $R_{c}$. As the bacterial host, we used $E$. coli strain SP427 (19), which expresses green fluorescent protein (GFP) constitutively. The GFP expression allowed us to visualize the intact cells in the colonies using fluorescence microscopy. We grew the host cells embedded in a thin soft-agar layer on plates containing rich medium, which prevents the cells from swimming and causes them to form compact, approximately spherical colonies. We then sprayed phage lysate onto these plates at different times after incubation, thereby implementing phage attacks on colonies of different sizes (Methods). We sprayed enough phages that all of the phage-sensitive cells would have been killed if the same phage-to-cell ratio had been mixed in a homogeneous liquid culture (details in SI Appendix, section 9).

Fig. 4 shows images of representative colonies at the time of addition of phage $\mathrm{P} 1_{\mathrm{vir}}$ and at $16 \mathrm{~h}$ of incubation after phage addition. Fig. 4, Left shows the dark-field images, whereas Fig. 4, Right shows the green fluorescence images, which visualize intact bacteria that contain GFP. The panel " $5 \mathrm{~h}$ " displays the rather small colony size that was typically obtained after $5 \mathrm{~h}$ of incubation. The " $5 \mathrm{~h}+16 \mathrm{~h}$ " panel (Fig. 4, Top) shows the typical final colony after an additional $16 \mathrm{~h}$ of incubation without exposure to phage. In this case, the colony is not only large

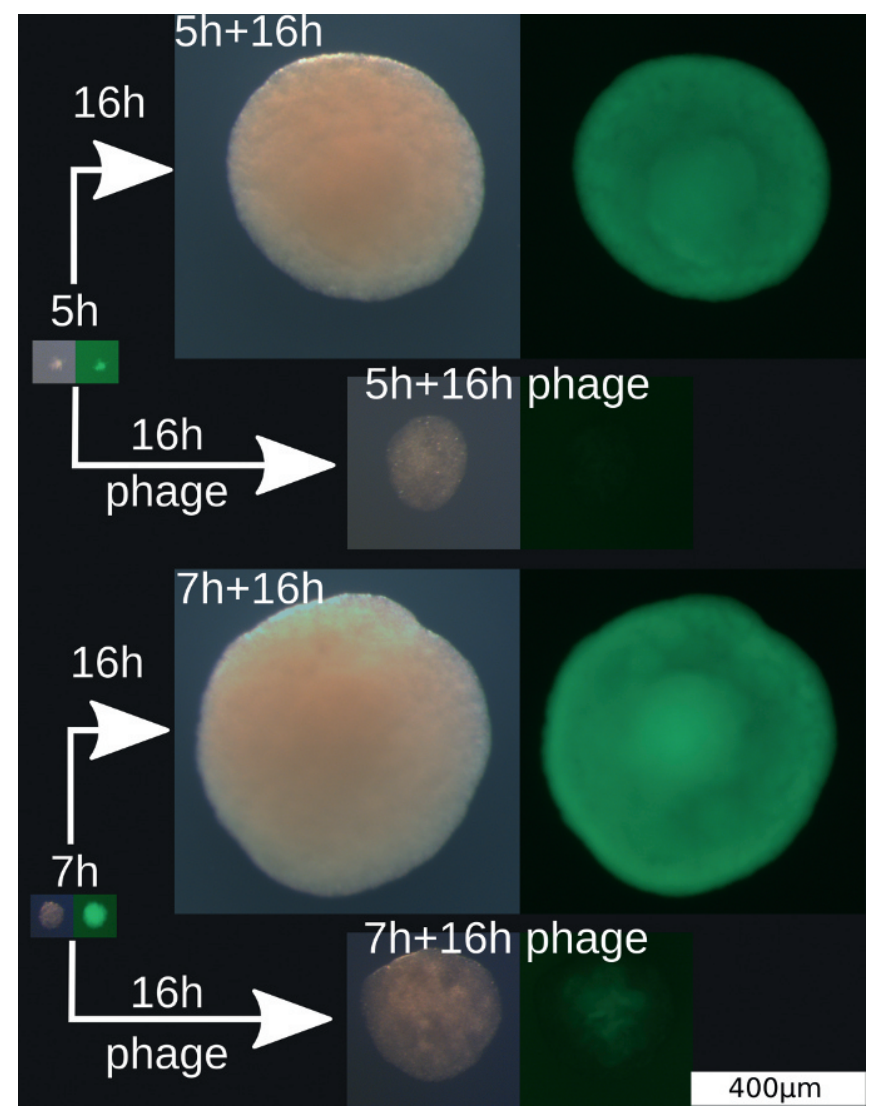

Fig. 4. Images of typical colonies before and after exposure to phages. The dark-field image and the corresponding green fluorescence image of the same colony are shown side by side. The green fluorescence images in the final colonies with phage exposure at $5 \mathrm{~h}$ and at $7 \mathrm{~h}$ spray time were collected at the same light source strength and with the same exposure time and are shown in the same magnification to allow direct comparison. Images of colonies that experienced different phage exposure times but identical total incubation times are shown in SI Appendix, Figs. S9 and $\mathrm{S} 10$. 

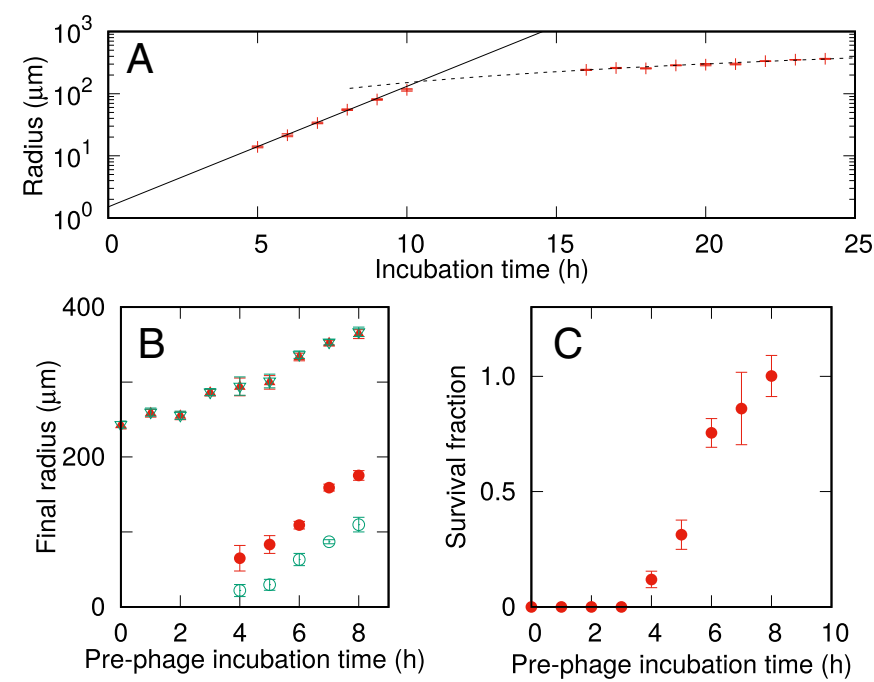

Fig. 5. $\mathrm{P} 1_{\text {vir }}$ infection of $E$. coli microcolonies. $(A)$ Growth in colony radius over time in the absence of phages. The solid line marks an exponential fit with a doubling time of $31 \mathrm{~min}$. The dashed line is a linear fit to growth in colony radius on longer timescales. The crossover point is $\sim 10 \mathrm{~h}$. The radius was estimated from the colony image from the area $A$ as $\sqrt{A / \pi}$. (B) Final colony size as a function of the incubation time before phage exposure. The solid red symbols show the estimates from dark-field images whereas the open green symbols show the radius estimated from the green fluorescence images. The triangles between $200 \mu \mathrm{m}$ and $400 \mu \mathrm{m}$ show the final radius of colonies in the absence of phages, while the circles show the final radius of colonies exposed to phages. The SEM is shown as error bars. (C) Number of visible colonies on plates sprayed with phages relative to the mean number of colonies on control plates without phages. The control plates had $76 \pm 4$ visible colonies per plate. The horizontal axis shows the microcolony growth time before the exposure to phages.

but also dense and homogeneously fluorescent. In contrast, the " $5 \mathrm{~h}+16 \mathrm{~h}$ phage" panel (Fig. 4, Upper Middle) shows a typical final colony when the plate has been sprayed with phage lysate after $5 \mathrm{~h}$ of growth and then incubated $16 \mathrm{~h}$ after that. One sees that the colony has grown bigger after incubation in the presence of phages, but it exhibits only faint fluorescence, indicating that most of the colony shown in the dark-field image consisted of dead remnants of bacteria.

The typical colony obtained after $7 \mathrm{~h}$ undisturbed growth is shown in the "7h" panel in Fig. 4, Bottom, and is visibly larger than the typical colony after $5 \mathrm{~h}$ of incubation, reflecting the additional four doublings of biomass. The panel " $7 \mathrm{~h}+16 \mathrm{~h}$ phage" shows the final size of this colony after it was exposed to phages and then incubated for 16 more hours. Although it is visibly smaller than the undisturbed colony in the " $7 \mathrm{~h}+16 \mathrm{~h}$ " panel, one observes a fluorescent region in the middle, suggesting a substantial amount of surviving bacteria.

Using microscopy images, we measured the growth of colonies that had not been exposed to phages and show that the radius grows exponentially (Fig. $5 A$ ). This growth is consistent with a doubling time of $30 \mathrm{~min}$ for about $9 \mathrm{~h}$ until a size of $2 \times 10^{6} \mathrm{bac}-$ teria if we assume a spherical colony of dense cells with volumes of $1 \mathrm{\mu m}^{3}$. After about $10 \mathrm{~h}$, the growth rate is reduced substantially and colony size eventually stabilizes at a level set by the initially available nutrients and the number of colonies per plate sharing those nutrients. Fig. $5 B$ shows how this final colony size varies moderately between experiments without phage exposure (triangles). The final radius of $400 \mu \mathrm{m}$ implies that our conditions allow colonies to reach $\sim 2 \times 10^{8}$ bacteria. Because slowgrowing bacteria are smaller than rapidly growing bacteria (20), we cannot accurately deduce the actual number of bacteria in these colonies.
Predictably, exposure to phages alters the colonies dramatically. Fig. $5 B$ (circles) shows the final size of colonies that were exposed to phages. In the dark-field recording we see a moderate change in the radius of final colonies from about $90 \mu \mathrm{m}$ to about $180 \mu \mathrm{m}$ as the phage exposure time is delayed. The corresponding change in size of the GFP-expressing part of the colony increases more dramatically from $20 \mu \mathrm{m}$ to about $100 \mu \mathrm{m}$. In all cases, the outer layers of the colony appear dark, indicating that the surfaces of these colonies consist of bacterial debris from $E$. coli that were killed by phages. Fig. $5 C$ quantifies the number of colonies per plate that are visible with the naked eye after the final incubation period for different prephage incubation times. The surviving fraction is normalized to the average number of visible colonies per control plate that were not exposed to phage. Almost no colonies had grown to visible size when the phages were introduced earlier than $4 \mathrm{~h}$ of incubation. The absence of surviving colonies was confirmed using fluorescence microscopy with $10 \times$ magnification. After this critical time, visible colonies emerged. When phages were introduced at $4 \mathrm{~h}$, a few small colonies could be detected. When phages were introduced at $6 \mathrm{~h}$ and later, almost $100 \%$ of the initial cells grew to form visible colonies. Thus, this laboratory experiment reproduced a steep transition in the number of surviving colonies that depended on the size of the microcolony at the time of phage addition. Two additional repetitions of the experiment confirmed the jump in colony survival when phages were introduced after 5-6 h of prephage incubation (SI Appendix, section 6).

An alternative explanation for the microcolony size-dependent jump in colony survival (Fig. $5 C$ ) could be that the jump reflects the critical colony size at which there is a large probability that the colony contains one or more mutated cells that have become phage resistant at the time of phage exposure and therefore can continue to grow and form a colony despite the killing of all of the phage-sensitive cells. Phage resistance and our spatial refuge model are not mutually exclusive but could both contribute to colony survival. To estimate the frequency of resistant bacteria in the surviving microcolonies, we picked cells from 50 of the microcolonies exposed to phages and restreaked them onto fresh plates to obtain "offspring" colonies formed by individual surviving cells in the presence of the chelator sodium citrate, which inactivates free phages and thereby permits colony formation by both sensitive and resistant cells. Up to 10 randomly picked offspring colonies from each original microcolony were then tested for resistance to $\mathrm{P} 1_{\text {vir }}$ by cross-streaking (see SI Appendix, Table S2 for complete results).

Table 1, "offspring," shows that sensitive and resistant cells were found in approximately equal numbers among the randomly picked offspring colonies arising from microcolonies that had been sprayed with phage after $4-8 \mathrm{~h}$ of prephage incubation. While these numbers demonstrate the expected strong selection pressure favoring phage-resistant mutants, which increased in frequency from $1.2 \times 10^{-5}$ in the starting culture to $\sim 0.5$ in the surviving colonies, they also demonstrate that many phagesensitive cells can survive the attack by $\mathrm{P} 1_{\mathrm{vir}}$. Importantly, when the offspring are grouped by the microcolony they originated

\section{Table 1. $\quad \mathbf{P} 1_{\text {vir }}$ resistance test summary}

\begin{tabular}{lccccccc} 
Time, h & \multicolumn{3}{c}{ "Offspring" } & \multicolumn{4}{c}{ Original microcolonies } \\
\hline & Sensitive & Resistant & Total & All sensitive & Mix & All resistant & Total \\
4 & 47 & 13 & 60 & 4 & 1 & 1 & 6 \\
5 & 22 & 10 & 32 & 4 & 0 & 1 & 5 \\
6 & 20 & 50 & 70 & 2 & 0 & 5 & 7 \\
7 & 41 & 45 & 86 & 4 & 1 & 4 & 9 \\
8 & 51 & 49 & 100 & 5 & 1 & 4 & 10 \\
Total & 181 & 167 & 348 & 19 & 3 & 15 & 37 \\
\hline
\end{tabular}


from (Table 1, original microcolonies), it is clear that no resistant cells were found in about half of the microcolonies from which we could recover live cells (19/37), suggesting that these colonies contained typically more than $90 \%$ phage-susceptible cells and therefore could not have survived due to protection by classical herd immunity. Furthermore, these susceptible colonies all grew substantially after exposure to phages, demonstrating that susceptible bacteria can dominate colony growth during prolonged phage exposure. We note that our inability to recover live cells from $13 / 50$ of the sampled microcolonies does not mean that all cells in those colonies were dead, since we recovered cells from the soft-agar-embedded colonies by sampling them with a toothpick rather than extracting whole colonies.

\section{Discussion}

This paper argues for a type of herd immunity associated with consecutive layers of bacteria that shield each other from infection. The modeled colonies exposed to phages at various time points in their growth predicted the existence of a minimal critical size of microcolony above which the inner growth overwhelms the killing by phages on the colony surface. We experimentally demonstrated that shielding can take place, by showing that $\mathrm{P} 1_{\mathrm{vir}}$-sensitive cells often constitute the majority of the survivors in growing $\mathrm{P} 1_{\text {vir }}$-infected colonies. Due to a strong selection pressure for $\mathrm{P} 1_{\text {vir }}$ resistance, at least in the outer layers of the microcolonies, phage-resistant mutants also accumulated in our experiments. The resistant mutants could protect phage-sensitive cells by herd immunity if they $(i)$ interfere with the spread of phages and (ii) constitute a greater fraction of the microcolony than the herd immunity threshold (21). The P1 $1_{\text {vir }}$-resistant mutants we analyzed (SI Appendix, section 8) and the ones reported in the literature $(22)$ all result in resistance because they abolish $\mathrm{P} 1_{\text {vir }}$ adsorption, meaning they are "invisible" to $\mathrm{P} 1_{\mathrm{vir}}$ and do not interfere with the spread. Further, from half of the surviving colonies, 10 of 10 randomly picked offspring were all phage sensitive, certainly suggesting a belowthreshold resistant fraction. Thus, while phage resistance predictably occurred in our experiments, it is unlikely to have played a significant role in the shielding of phage-sensitive cells in these microcolonies.

Experimentally, the critical microcolony growth time is not as sharp as in the numerical simulation. This is expected because the vertical position of the microcolonies varies within the $\sim 0.4$-mm-thick top-agar layer, and the colonies at the bottom will be exposed to phages later than those nearer the top. We confirmed the jump of the survival fraction to almost $100 \%$ at around $6 \mathrm{~h}$ prephage incubation time in additional experiments where we varied the soft-agar thickness (SI Appendix, section 6). The additional time needed for diffusion through the top agar brings a conservative estimate of the actual critical phage arrival time to about $6-7 \mathrm{~h}$, resulting in an estimated $R_{c} \approx 25 \mu \mathrm{m}$ critical microcolony radius. Image analysis confirmed that each final colony had grown significantly beyond this size, supporting the proposed survival scenario. Quantitatively, this $R_{c}$ value is three to five times larger than the typical results of the simulation in Fig. 3. Part of this disagreement could be because we simplified the cell shape to be a sphere in the simulations, while E. coli is known to have an elongated rod shape. If the long edge of a cell is pointed vertically into the microcolony, as is apparently the norm for other rod-shaped bacteria (23), the burst of such a cell allows phages to penetrate significantly deeper than in our scenario. Thereby the elongated shape of $E$. coli increases the penetration depth $\Delta R$ and hence increases $R_{c}$ proportionally.

The fate of a colony may not only depend on whether its growth can outrival the lysis on the surface. In particular, when bacteria grow slower, phage infections tend to yield smaller burst sizes with longer latency times $(24,25)$ and most phages cease propagation on stationary-phase cells (26). This means that a colony which approaches its resource-limited size may survive a phage attack simply because the phages cannot propagate. In our study, this effect was not important for the determination of the critical size, since the threshold for colony survival was at most $7 \mathrm{~h}$, and exponential growth continued past the 9-h mark. Thus, the surviving colonies had certainly grown after encountering the phages.

Since our proposed mechanism does not rely on specific molecular components in either the host or the phage, we believe our findings will generalize to a broad range of bacterium-phage pairs, a prediction which should be experimentally tested. Our model further predicts that protection will be limited in special cases where the latency time is very short and/or the adsorption rate is small. A similar effect of adsorption rate was observed for $\lambda$ phages infecting bacteria in biofilm (27), indicating the importance of optimization of adsorption rate for phages to spread in a spatially structured habitat. Finally, the survival of sensitive cells after overnight incubation in the presence of phages depends on the inability of phages to propagate on nongrowing cells. Therefore, we predict that phages which can continue killing of stationary-phase cells [e.g., T7 (14)] would not favor colony survival.

Theoretical models supplemented with experiments on phagebacteria culture in liquid media demonstrated $(1,2,16)$ that bacteria and virulent phages persistently can coexist. They do so in self-organized critical conditions where only one phage from each bacterial lysis event persists to infect another susceptible host. Our present study suggests to view this coexistence differently when dealing with bacteria growing in a gel or a semisolid, as for example in soil or even in submillimeter scale in ocean water (28). Because all new bacteria are generated from bacteria that are already present, the spatial distribution of the host will be maintained over long time periods. The destiny of released phages then becomes crucially dependent on the distances to new colonies of hosts, and the overall survival of a virulent phage may then rely on "farming" the local colony in a sustainable way.

The observation of bacterial colonies with an active core surrounded by an extended region of likely dead (nonfluorescent) bacteria suggests yet another possibility that may enhance colony robustness. As dead bacteria accumulate on the surface of the colony, phage particles may lose their DNA by injecting it into these hosts' remnants. Thus, the debris may cause a sink for phage DNA at the surface of the colony that further enhances survival in its core.

In any case, our paper suggests that the evolution of especially long latency time and high adsorption rate for a virulent phage may reflect its ability to live in a persistently infectious parasitic state on a growing bacterial colony. A related persistent infection pattern is seen in filamentous phages (e.g., M13) that, on the scale of the individual bacteria, maintains the host alive while producing phages (29). In contrast, phages with small adsorption rates and short latency times will more easily exterminate a colony. One outcome of our colony-focused "microcosmos" is thus that the "choice" of latency time for a phage is more than just an optimization between fast generations with few offspring and longer generations with much larger burst size (30). For lytic phages, the art of killing (12) has more facets than growth on a well-mixed host population.

\section{Methods}

Numerical Simulation. The motion of the cells and phage is modeled without inertia. The cells are modeled as 3D spheres that repel each other when they overlap (31). An uninfected cell grows exponentially with a nutrientdependent rate and divides into two cells when it reaches a threshold volume. The median of the cell volume is set to be $1.3 \mu \mathrm{m}^{3}$. We assume nutrients are spatially homogeneous and consumed as the cells grow. A phage is modeled as a point particle that undergoes diffusion. When the phage 
overlaps with a cell, a repulsive force is exerted from the cell. A phage overlapping with a cell is adsorbed with a rate $\gamma$. When a cell is infected by a phage, it produces phage particles after an average latency time $\tau_{L}$ (13). During the latency period, an infected cell can adsorb more phages. When a lysis occurs, $\beta$ new phage particles are spawned uniformly around the cell's center and the cell is removed. The simulation starts with a single cell, which is allowed to establish a colony for the time duration $T_{i}$. At time $T_{i}$, phages are spawned uniformly in the simulated volume outside of the colony. Details are in SI Appendix, section 1. The code is available as Dataset S1.

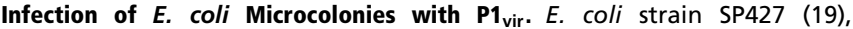
an MC4100 derivative containing a chromosomally encoded $P_{\mathrm{A} 1 / 04 / 03}::$ gfpmut3b gene cassette (32), was grown overnight in LB (33) supplemented with $50 \mu \mathrm{g} / \mathrm{mL}$ kanamycin. The culture was diluted to $\sim 400 \mathrm{cfu} / \mathrm{mL}$ in sterile MC buffer $\left(50 \mathrm{mM} \mathrm{CaCl}_{2}, 25 \mathrm{mM} \mathrm{MgCl}\right.$ ) and kept at room temperature. Every hour, $250 \mu \mathrm{L}$ of the diluted culture was mixed with $2.5 \mathrm{~mL}$ R-top agar (as in ref. 34 but with $5 \mathrm{~g} / \mathrm{L}$ agar and $50 \mu \mathrm{g} / \mathrm{mL}$ kanamycin), plated on fresh R agar plates (as R top but with $12 \mathrm{~g} / \mathrm{L}$ agar), and incubated at $37^{\circ} \mathrm{C}$ to allow individual cells to form microcolonies. After a given incubation

1. Campbell A (1961) Conditions for the existence of bacteriophage. Evolution 15: 153-165.

2. Levin BR, Stewart FM, Chao L (1977) Resource-limited growth, competition, and predation: A model and experimental studies with bacteria and bacteriophage. Am Nat 111:3-24.

3. Lenski RE (1988) Dynamics of interactions between bacteria and virulent bacteriophage. Adv Microb Ecol 10:1-44.

4. Heilmann S, Sneppen K, Krishna S (2012) Coexistence of phage and bacteria on the boundary of self-organized refuges. Proc Natl Acad Sci USA 109:12828-12833.

5. Jover LF, Cortez MH, Weitz JS (2013) Mechanisms of multi-strain coexistence in hostphage systems with nested infection networks. J Theor Biol 332:65-77.

6. Haerter JO, Mitarai N, Sneppen K (2014) Phage and bacteria support mutual diversity in a narrowing staircase of coexistence. ISME $J$ 8:2317-2326.

7. Stewart FM, Levin BR (1984) The population biology of bacterial viruses: Why be temperate. Theor Popul Biol 26:93-117.

8. Maslov S, Sneppen K (2015) Well-temperate phage: Optimal bet-hedging against local environmental collapses. Sci Rep 5:10523.

9. De Paepe M, Taddei F (2006) Viruses' life history: Towards a mechanistic basis of a trade-off between survival and reproduction among phages. PloS Biol 4:e193.

10. Demerec M, Fano U (1945) Bacteriophage-resistant mutants in Escherichia coli. Genetics 30:119-136.

11. Hershey AD (1946) Mutation of bacteriophage with respect to type of plaque. Genetics 31:620-640.

12. Tzu S(ca. 450 BC) Sun Tzu on the Art of War: The Oldest Military Treatise in the World, Translated from the Chinese with Introduction and Critical Notes, trans Giles L (1910) (Luzac \& Company, London).

13. Mitarai N, Brown S, Sneppen K (2016) Population dynamics of phage and bacteria in spatially structured habitats using phage $\lambda$ and Escherichia coli. J Bacteriol 198: 1783-1793.

14. Abedon S, Yin J (2008) Impact of spatial structure on phage population growth. Bacteriophage Ecology, ed Abedon S (Cambridge Univ Press, Cambridge, UK), Vol 15, pp 94-113.

15. Shao X, et al. (2017) Growth of bacteria in 3-d colonies. PLoS Comput Biol 13:e1005679.

16. Thingstad TF (2000) Elements of a theory for the mechanisms controlling abundance, diversity, and biogeochemical role of lytic bacterial viruses in aquatic systems. Limnol Oceanogr 45:1320-1328.

17. Taylor BP, Penington CJ, Weitz JS (2017) Emergence of increased frequency and severity of multiple infections by viruses due to spatial clustering of hosts. Phys Biol 13:066014. time at $37{ }^{\circ} \mathrm{C}, 0.5 \mathrm{~mL}$ of $\mathrm{P} 1_{\text {vir }}$ phage lysate $\left(7 \times 10^{9} \mathrm{pfu} / \mathrm{ml}\right)$ was sprayed uniformly on the top agar surface, using a perfume bottle with an atomizer bulb (35), and the plate was incubated for another $16 \mathrm{~h}$ at $37{ }^{\circ} \mathrm{C}$. One control plate without the addition of $\mathrm{P} 1_{\text {vir }}$ was processed along with triplicate phage-spray plates for each time point. The experiment was repeated three times with similar results. Details of the repeat experiments can be found in SI Appendix, section 6. Microscopy images were obtained using a Leica MZ16F fluorescence stereomicroscope and quantification was done by matlab code developed by the authors. The peripheral $1 \mathrm{~cm}$ of each plate was excluded in the analysis to avoid any effects of heterogeneity in phage spraying close to the edge. Six additional plates without phage addition were prepared as the control plates above and used for microscopy imaging to determine microcolony size from 5-10 h of incubation at $37^{\circ} \mathrm{C}$.

ACKNOWLEDGMENTS. The authors sincerely thank Stanley Brown for help ful discussions and Linda Hove Christensen for technical assistance. This work was funded by the Danish National Research Foundation (BASP: DNRF120) and the European Research Council under the European Union's Seventh Framework Programme (FP/2007 2013)/ERC Grant Agreement 740704.

18. Ikeda H, Tomizawa Ji (1965) Transducing fragments in generalized transduction by phage p1: I. Molecular origin of the fragments. J Mol Biol 14:85-109.

19. Bahl MI, Sorensen SJ, Hansen LH (2004) Quantification of plasmid loss in Escherichia coli cells by use of flow cytometry. FEMS Microbiol Lett 232:45-49.

20. Bremer H, Dennis PP (1996) Modulation of chemical composition and other parameters of the cell by growth rate. Escherichia coli and Salmonella, ed Neidhardt FC (ASM Press, Washington, DC), pp 1553-1569.

21. Payne P, Geyrhofer L, Barton NH, Bollback JP (2017) CRISPR-based herd immunity limits phage epidemics in bacterial populations. bioRxiv:10.1101/181487.

22. Franklin NC (1969) Mutation in galu gene of $E$. coli blocks phage p1 infection. Virology 38:189-191.

23. Drescher $\mathrm{K}$, et al. (2016) Architectural transitions in Vibrio cholerae biofilms at singlecell resolution. Proc Natl Acad Sci USA 113:E2066-E2072.

24. Cohen SS (1949) Growth requirements of bacterial viruses. Bacteriol Rev 13: $1-24$.

25. Weitz JS, Dushoff J (2008) Alternative stable states in host-phage dynamics. Theor Ecol 1:13-19.

26. Abedon ST, Yin J (2009) Bacteriophage plaques: Theory and analysis. Bacteriophages: Methods and Protocols: Isolation, Characterization, and Interactions, eds Clokie MRJ, Kropinski A (Humana Press, New York), Vol 1, pp 161-174.

27. Gallet R, Shao Y, Wang N (2009) High adsorption rate is detrimental to bacteriophage fitness in a biofilm-like environment. BMC Evol Biol 9:241.

28. Azam F, Malfatti F (2007) Microbial structuring of marine ecosystems. Nat Rev Microbiol 5:782-791.

29. Kehoe JW, Kay BK (2005) Filamentous phage display in the new millennium. Chem Rev 105:4056-4072.

30. Abedon ST, Herschler TD, Stopar D (2001) Bacteriophage latent-period evolution as a response to resource availability. Appl Environ Microbiol 67:42334241.

31. Mitarai N, Jensen MH, Semsey S (2015) Coupled positive and negative feedbacks produce diverse gene expression patterns in colonies. mBio 6:e00059-15.

32. Normander B, Christensen BB, Molin S, Kroer N (1998) Effect of bacterial distribution and activity on conjugal gene transfer on the phylloplane of the bush bean (Phaseolus vulgaris). Appl Environ Microbiol 64:1902-1909.

33. Bertani G (1951) Studies on lysogenesis. I. The mode of phage liberation by lysogenic Escherichia coli. J Bacteriol 62:293-300.

34. Miller JH (1972) Experiments in Molecular Genetics (Cold Spring Harbor Lab Press, Cold Spring Harbor, NY)

35. Schedl P, Primakoff P (1973) Mutants of Escherichia coli thermosensitive for the synthesis of transfer RNA. Proc Natl Acad Sci USA 70:2091-2095. 